\title{
Expanded Prostate Cancer Index Composite, Sexual Medications/Devices Supplement
}

National Cancer Institute

\section{Source}

National Cancer Institute. Expanded Prostate Cancer Index Composite, Sexual

Medications/Devices Supplement. NCI Thesaurus. Code C107631.

A component of the EPIC designed to assess the use of erectile aids among patients treated for prostate cancer. 